\title{
DÜBLIN
}

Technological University Dublin

ARROW@TU Dublin

\section{Kinetic Studies for the Preparation of Probiotic Cabbage Juice: Impact on Phytochemicals and Bioactivity}

\author{
Amit Jaiswal \\ Technological University Dublin, amit.jaiswal@tudublin.ie \\ Nissreen Abu-Ghannam \\ Technological University Dublin, nissreen.abughannam@tudublin.ie
}

Follow this and additional works at: https://arrow.tudublin.ie/schfsehart

Part of the Food Biotechnology Commons, Food Chemistry Commons, Food Microbiology Commons, and the Food Processing Commons

\section{Recommended Citation}

Jaiswal, A. K. \& Abu-Ghannam, N. (2013). Kinetic studies for the preparation of probiotic cabbage juice: Impact on phytochemicals and bioactivity. Industrial Crops \& Products, 50(0), 212-218. Doi : 10.1016/ j.indcrop. 2013.07.128

This Article is brought to you for free and open access by the School of Food Science and Environmental Health at ARROW@TU Dublin. It has been accepted for inclusion in Articles by an authorized administrator of ARROW@TU

Dublin. For more information, please contact arrow.admin@tudublin.ie, aisling.coyne@tudublin.ie, gerard.connolly@tudublin.ie.

Funder: Irish Government under the Technological Sector Research Scheme (Strand III) of the National Development Plan.

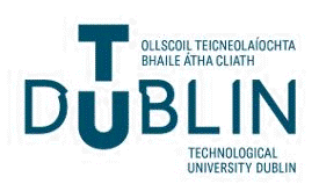




\section{Highlights}

- Kinetic studies for the preparation of probiotic cabbage juice were carried out.

- Significant growth in LAB was observed in white cabbage juice.

- The Gompertz model was applied to predict probiotic growth in white cabbage juice.

- Lactic acid was the major end product of the fermented cabbage juice.

- LAB fermented cabbage juice retains more than $75 \%$ of phytochemicals and bioactivity. 
Kinetic studies for the preparation of probiotic cabbage juice: Impact on phytochemicals and bioactivity

\section{Amit Kumar Jaiswal \& Nissreen Abu-Ghannam*}

School of Food Science and Environmental Health, College of Sciences and Health, Dublin Institute of Technology, Cathal Brugha Street, Dublin 1, Ireland

Short running head: Kinetic studies on probiotic cabbage juice preparation

*Corresponding author: Tel: +353-1-402-7570; Fax: +353-1-878-8978

E-mail: nissreen.abughannam@ dit.ie (Nissreen Abu-Ghannam); amit.jaiswal@ dit.ie (Amit Kumar Jaiswal) 


\begin{abstract}
A kinetic study for the production of probiotic cabbage juice was carried out under controlled $\mathrm{pH}$ and dissolved oxygen using several strains of Lactic acid bacteria (LAB). Furthermore, effect of probiotic fermentation on polyphenolic content and antioxidant capacity was investigated. Results showed significant growth in Lactobacillus brevis, Lactobacillus plantarum and Lactobacillus rhamnosus corresponding to $9.19,9.47$ and $10.6 \log \mathrm{CFU} / \mathrm{ml}$ after $24 \mathrm{~h}$ of fermentation, which is satisfying criteria for a food product to be characterized as probiotic. Lactic acid (LA) was the major end product of the fermented cabbage juice attaining the concentrations of 6.97, 9.69 and $12.2 \mathrm{~g} / \mathrm{LA}$ for L. plantarum, L. rhamnosus and L. brevis, respectively. LAB fermentation retains more than $75 \%$ of total phenolic content (TPC) and total flavonoid content (TFC) of the initial raw material, and similar set of results were observed for antioxidant capacity. First-order kinetics model fitted well with the experimental data with $\mathrm{R}^{2}$ value ranging from 0.92-0.96, 0.96-0.98 and 82.2-97.2 for TPC, TFC and antioxidant capacity, respectively. During refrigerated storage $\left(4^{\circ} \mathrm{C}\right)$, all the probiotic cultures met the criterion of maintaining counts greater than $8 \log \mathrm{CFU} / \mathrm{ml}$; in addition to maintaining bioactive components and antioxidant capacity.
\end{abstract}

Keywords: Antioxidant capacity; Kinetic study; Lactic acid; Polyphenols; Probiotics; cabbage juice 


\section{Introduction}

Foods which promote health beyond providing basic nutrition are termed as 'functional foods'. These foods have the potential to promote health in ways not anticipated by traditional nutritional science (Beganović et al., 2011). Modern consumers are showing continuously a remarkable interest in functional foods with more emphasis in recent times on probiotic types of products. The main motive for purchasing functional foods is the growing desire to use foods either to help prevent chronic illnesses or to optimize health. During the past two decades there has been a large increase in the worldwide sales of functional products containing probiotic bacteria which led to one of the fastest growing food sectors, with a compound annual growth rate of $8.6 \%$ in the 10 years to 2012 (Khan et al., 2013).

Available literature confirms that the addition of probiotics to food provides several health benefits, including reduction in the level of serum cholesterol, improved gastrointestinal function, enhanced immune system, antimutagenic property, anti-carcinogenic property, antidiarrheal property, improvement in inflammatory bowel disease and suppression of Helicobacter pylori infection by the addition of selected strains to food products (Agerholm-Larsen et al., 2000; Gotcheva et al., 2002; Nomoto, 2005; Sindhu and Khetarpaul, 2003).

Probiotics have successfully been added to a wide range of dairy based food products. However, the problem of lactose intolerance and cholesterol content has increased the demand for nondairy based probiotic products. About 5 to $15 \%$ of the Europe population is lactose intolerant and this number increased up to $80 \%$ in some part of the world such as central Asia and Africa (de Vrese et al., 2001). Dairy products with probiotic bacteria are unsuitable for this group of population because of its health condition. Additionally, with growing awareness of gut health, 
consumers demand a wider variety of probiotic products beyond dairy based food products. This work is part of an on-going project to evaluate the potential of Brassica vegetables for the development of a probiotic-based product. Fermentation is widely used in the food industry to improve the sensory characteristics of a product as well as to eliminate certain undesirable constituents, make nutrients more accessible while preserving and even improving the nutritional properties. In a previous study, it was reported that cabbage juice is a good medium for the growth of probiotics (Yoon et al., 2006). It was also observed that natural fermentation of cabbage in the production of sauerkraut increased the initial antioxidant (AO) activity which could have resulted from the combined effects of wounding and chemical processes incurred by lactic acid bacteria (LAB) (Kusznierewicz et al., 2008).

Furthermore, there is now an increasing interest in modelling the kinetics of beneficial microorganisms in food systems leading to a better understanding of the fermentation process. Mathematical models can help to predict the influence of fermentation operating parameters on the rate of substrate utilization, cell growth and lactic acid (LA) production (Biazar et al., 2003). The use of these models may lead to the development of better strategies for the optimization of the fermentation process to ensure its economical viability. Therefore, in the present report, a kinetic study for the production of probiotic cabbage juice was carried out using several strains of $\mathrm{LAB}$ in order to achieve maximum $\mathrm{LAB}$ in cabbage juice. At the same time, the effect of fermentation on inherent total phenolic content (TPC), total flavonoid content (TFC), and AO capacity of cabbage juice was also studied. Furthermore, shelf life of fermented probiotic cabbage juice was undertaken by evaluating the cell viability, lactic acid content, $\mathrm{pH}$ and phytochemical constituents. 


\section{Materials and methods}

\subsection{LAB strains and inoculum preparation}

L. plantarum ATCC 8014; Lactobacillus rhamnosus ATCC 9595 and Lactobacillus brevis ATCC 8287 were purchased from Medical Supply Company, Dublin, Ireland. The culture was maintained at $-70^{\circ} \mathrm{C}$ in $20 \%$ glycerol stocks and grown in de Man, Rogosa and Sharp (MRS) broth (Scharlau Chemie, Barcelona, Spain) at $37^{\circ} \mathrm{C}$. For the preparation of inoculum, $25 \mathrm{ml}$ of sterile MRS broth was inoculated with $1 \mathrm{ml}$ of thawed stock culture and incubated at $37^{\circ} \mathrm{C}$ for 12-14 h. This was then serially diluted 100 times to obtain working culture containing 5-6 $\log$ colony-forming unit $(\mathrm{CFU}) / \mathrm{ml}$ cells as determined by plate counts.

\subsection{Plant materials and preparation of juice}

Fresh white cabbage (Brassica oleracea var. capitata) was purchased from a local supermarket in Dublin. Twenty five to thirty white cabbage heads $(45-50 \mathrm{~kg})$ were randomly selected and trimmed of their outer leaves and the stem. The heads were then divided into four segments, and the central core was removed. The segments were chopped into small pieces using an ordinary knife. The part of shredded cabbage was blended with the addition of water $(1: 1 \mathrm{w} / \mathrm{v})$, and the juices were squeezed out from the pulps and sterilized for $15 \mathrm{~min}$ at $121^{\circ} \mathrm{C}$ in an autoclave (Tomy SS-325, Tomy Seiko Co. Ltd, Tokyo, Japan) and stored under dark refrigerated conditions $\left(4^{\circ} \mathrm{C}\right)$. The sterilized juice was filtered through sterilized muslin cloth and further diluted with sterile double distilled water $(2: 1 \mathrm{v} / \mathrm{v})$ (hereafter the diluted cabbage juice was called WCJ ) and five litre of WCJ was used for each batch of fermentation. 


\subsection{Preliminary LAB fermentation}

In order to check the applicability of WCJ as a substrate for LAB growth and to compare the growth pattern in WCJ with typical medium for LAB cultures, preliminary experiments involved screening of WCJ (diluted with water, 2:1) and MRS broth. The preliminary LAB fermentation was performed in a 96-well round-bottom microplate (Sarstedt, Inc, USA). The sterilized WCJ was inoculated with various LAB strains $(5 \% \mathrm{v} / \mathrm{v})$, and MRS was inoculated in similar fashion and $200 \mu \mathrm{l}$ dispensed in each microtiter well. Wells containing sterile MRS or WCJ (200 $\mu \mathrm{l})$ were treated as blanks to check for contamination. The LAB growth was monitored at $600 \mathrm{~nm}$ using the micro plate spectrophotometer (Powerwave, Biotek, VT, USA) (preceded with $30 \mathrm{sec}$ agitation) over $24 \mathrm{~h}$ at $30 \mathrm{~min}$ intervals. Growth curves of the test organisms were analyzed graphically as a plot of $\mathrm{OD}_{600}$ versus time. Maximum $\mathrm{OD}_{600}\left(\mathrm{OD}_{\max }\right)$ obtained and lag time $(\lambda)$ for each growth curve were calculated using Gen5 reader data analysis software.

\subsection{Fermentation in $7 \mathrm{~L}$ bioreactor under controlled $\mathrm{pH}$}

Seed culture $(200 \mathrm{ml})$ was prepared as mentioned in section 2.1 (LAB strains and inoculum preparation). Cultivation was carried out at $37^{\circ} \mathrm{C}$ at the agitation speed of $200 \mathrm{rpm}$, in a 7 litre Bioflo 415 bioreactor (New Brunswick Scientific Ltd.) containing 5 litre of WCJ under aseptic conditions. The bioreactor was sterilized in situ, cooled and then inoculated with 5\% inoculum (v/v). Culture $\mathrm{pH}$ was maintained at 7.0 by the automated control system of bioreactor with the addition of acid or base. Samples were withdrawn at 3-4 h interval and analyzed for viable cell count, lactic acid (LA) production, phytochemical constituents and AO capacity. 


\subsection{Viable cell counts}

Viable cell counts in the fermented WCJ $(\log \mathrm{CFU} / \mathrm{ml})$ were determined by the standard plate method with MRS medium. Dilution of $1 \mathrm{ml}$ broth was carried out in $9 \mathrm{ml}$ maximal recovery diluent (MRD) to plate the suitable dilution. The plates were incubated at $37^{\circ} \mathrm{C}$ for $36-48 \mathrm{~h}$, for cell enumeration.

\subsection{Effect of cold storage on probiotic WCJ}

After $24 \mathrm{~h}$ of fermentation at $37^{\circ} \mathrm{C}$, the fermented WCJ was stored at $4^{\circ} \mathrm{C}$ for four weeks. Samples were taken at three-day intervals, and $\mathrm{pH}$, lactic acid, viable cell count, phytochemical content and AO capacity were estimated.

\subsection{Analytical procedure}

Each sample of the fermented broth was centrifuged at $10,000 \mathrm{rpm}$ for $15 \mathrm{~min}$ at $4^{\circ} \mathrm{C}$. The supernatant was used for the analysis.

\subsubsection{Total phenolic and flavonoid contents}

TPC and TFC of samples were estimated according to our earlier report (Jaiswal et al., 2012b). In brief, for the TPC estimation, $100 \mathrm{ml}$ aliquot of sample in deionized water were mixed with 2 $\mathrm{ml}$ of $2 \% \mathrm{Na}_{2} \mathrm{CO}_{3}$ and were allowed to stand for $2 \mathrm{~min}$ at room temperature. After incubation, $100 \mathrm{ml}$ of $1 \mathrm{~N}$ Folin-Ciocalteau's phenol reagent was added. Reaction mixture was allowed to stand for $30 \mathrm{~min}$ at room temperature in the dark. Absorbance of all the sample solutions was measured at $720 \mathrm{~nm}$ using spectrophotometer (Genesys 20; Thermo Spectronic, Madison, WI). Results were expressed as $\mu \mathrm{g}$ gallic acid equivalents (GAE) per ml of sample.

For the TFC estimation, $250 \mathrm{ml}$ of sample was mixed with $1.25 \mathrm{ml}$ of deionized water and $75 \mathrm{ml}$ of $5 \% \mathrm{NaNO}_{2}$ solution. After $6 \mathrm{~min}, 150 \mathrm{ml}$ of $10 \% \mathrm{AlCl}_{3} \cdot \mathrm{H}_{2} \mathrm{O}$ solution was added. Finally, 0.5 
$\mathrm{ml}$ of $\mathrm{NaOH}(1 \mathrm{M})$ solution was added and the total volume was made up to $2.5 \mathrm{ml}$ with deionized water. Absorbance against blank was taken at $510 \mathrm{~nm}$ using a spectrophotometer. Results were expressed as $\mu \mathrm{g}$ quercetin equivalents $(\mathrm{QE})$ per $\mathrm{ml}$ of sample.

\subsubsection{Determination of sugar, organic acids and protein contents}

Determination of individual sugar content and organic acids was carried out as described in our earlier report (Jaiswal et al., 2012a). Standards for the organic acids such as lactic, propionic, citric, oxalic and acetic acid; sugars such as glucose, fructose and arabinose were used to identify and quantify the contents in the samples. Protein concentration of probiotic cabbage juice was estimated using Bradford's method (Bradford, 1976). In brief, $200 \mu$ l aliquot of sample was mixed with $800 \mu \mathrm{l}$ of Bradford's reagent (Sigma-Aldrich, Germany) and allowed to stand for 5 min at room temperature. Absorbance of all the sample solutions was measured at $595 \mathrm{~nm}$ using a spectrophotometer (Genesys 20; Thermo Spectronic, Madison, WI). Results were expressed as $\mu \mathrm{g}$ bovine serum albumin equivalents (BSAE) per $\mathrm{ml}$ of sample.

\subsubsection{Antioxidant capacity}

Two different methods [2,2-Diphenyl-1-picrylhydrazyl radical scavenging capacity (DPPH) and Ferric reducing AO potential (FRAP) assay were used for the estimation of AO capacity of the sample (Rajauria et al., 2012). DPPH scavenging capacity assay was performed in a 96-well round-bottom microplate with 1:1 ratio of $100 \mu$ of 2,2-Diphenyl-1-picrylhydrazyl (DPPH) (Sigma-Aldrich, Germany) radical solution $(165 \mu \mathrm{M})$ and $100 \mu \mathrm{l}$ of test sample. The DPPH solution was freshly prepared in methanol. The reaction mixtures were incubated for $30 \mathrm{~min}$ at $25^{\circ} \mathrm{C}$ in dark conditions, and absorbance measured at $517 \mathrm{~nm}$ in a microplate reader (Biotek 
EL808 Microplate Reader, BioTek Instruments, Inc., VT 05404, USA). Results were expressed as $\mu \mathrm{g}$ ascorbic acid equivalents (AscE) per $\mathrm{ml}$ of sample.

For FRAP analysis, reagent was freshly prepared by mixing 10 volumes of $300 \mathrm{mM}$ acetate buffer, pH 3.6, with 1 volume of $10 \mathrm{mM}$ TPTZ (2,4,6-tri(2-pyridyl)-s-triazine) (Sigma-Aldrich, Germany) in $40 \mathrm{mM}$ hydrochloric acid and with 1 volume of $20 \mathrm{mM}$ ferric chloride (SigmaAldrich, Germany). All solutions were used on the day of preparation. The reaction was performed in a microplate reader with 96-well plates (Sarstedt, Inc, USA) at a temperature of $37^{\circ} \mathrm{C}$. Preheated $100 \mu \mathrm{l}$ FRAP reagent at $37^{\circ} \mathrm{C}$ was dispensed in each well with $50 \mu \mathrm{l}$ of sample or standard. The reagent blank assay was performed by using $100 \mu 1$ FRAP reagent and $50 \mu 1$ of water. The absorbance was read after $10 \mathrm{~min}$ at $593 \mathrm{~nm}$ with the help of microplate spectrophotometer. Trolox (Sigma-Aldrich, Germany) was used as a standard and the results were expressed as $\mu \mathrm{g}$ trolox equivalents (TE) per ml of sample.

\subsection{Mathematical modelling}

Modified Gompertz equation (Zwietering et al., 1990) was fitted to the logarithm of the cell concentration in order to estimate the maximum specific growth rates of L. plantarum, $L$. rhamnosus and L. brevis. The model is described by the equation (Eq. 1):

$$
\log \left(\frac{N}{N_{0}}\right)=A \times \exp \left\{-\exp \left[\frac{\mu \times e}{A}(\lambda-t)+1\right]\right\}
$$

where $\mu_{\max }$ is the maximum specific growth rate of cell population, $\mathrm{A}$ is the log increase in population and $\lambda$ is a delayed time variable (lag phase), $\mathrm{N}$ is the CFU/ml at any time $\mathrm{t}, \mathrm{N}_{0}$ is the initial CFU/ml. 
The fermentative production of LA was described by the following equation (Eq. 2) (Mercier et al., 1992).

$$
\frac{d P}{d t}=P_{r} \times P\left(1-\frac{P}{P_{m}}\right)
$$

Where $\mathrm{t}$ is time, $\mathrm{P}$ is LA concentration $(\mathrm{g} / \mathrm{l}), \mathrm{Pm}$ is the maximum concentration of lactic acid $(\mathrm{g} / \mathrm{l}), \operatorname{Pr}\left(\mathrm{h}^{-1}\right)$ is the ratio between the initial volumetric rate of product formation (rp) and the initial product concentration $\mathrm{P}_{0}$. Eq. (2) can be solved to give the following expression:

$$
P=\frac{P_{0} P_{m} e^{P_{r} t}}{P_{m}-P_{0}+P_{0} e^{P_{r} t}}
$$

The value of model parameters for $\left(\mathrm{A}, \mu, \lambda . \mathrm{P}_{0}, \mathrm{Pm}\right.$ and $\left.\mathrm{Pr}\right)$ were fitted by with a Marquardt algorithm, using the STATGRAPHICS Centurion XV (StatPoint Technologies, Inc., Warrenton, VA) statistical software.

The kinetics of WCJ phenolic content, flavonoid content and different AO capacity were described by fitting a zero order (Eq. 4), first-order (Eq. 5) or a the second order kinetic model (Eq. 6) to the experimental data

$$
\begin{gathered}
A=A_{0} \pm k t \\
A=A_{0} \times \exp ( \pm k t) \\
\frac{A}{A}=\frac{A}{A_{0}} \pm k t
\end{gathered}
$$

Where, $\mathrm{A}$ is the parameter to be estimated, the sub index 0 indicates the initial value of the parameter, $\mathrm{t}$ is the fermentation time, and $k$ is the rate constant at temperature $\mathrm{T}$. For the 
parameter estimation, the individual measured concentrations were used instead of mean values of duplicate or triplicate experiments, thus taking into account variability within the samples.

\subsection{Statistical analysis}

Fermentation was carried out in duplicate whereas all the other analysis were carried out in triplicate and replicated twice unless otherwise stated. Results were expressed as mean values \pm standard deviation. The regression analysis and analysis of variance (ANOVA) were carried out using the STATGRAPHICS Centurion XV software. Values of $P<0.05$ were considered as statistically significant. The coefficient of determination $\left(\mathrm{R}^{2}\right)$ and mean square error (MSE) were used as criteria for adequacy of fit.

\section{Results and discussion}

\subsection{Initial fermentation studies with microtiter plate reader}

Prior to the application of bioreactor fermentation using 5 litre of WCJ, preliminary fermentation was carried out to check the applicability of WCJ as a substrate for LAB growth. LAB strains have complex nutritive requirements, and therefore, they are usually associated with nutrient-rich environments such as animal bodies, plants, and foodstuffs. LAB grew well in the WCJ without additional supplements, suggesting that WCJ comprises a good medium for the growth of LAB strains.

The $\mathrm{OD}_{\max }$ obtained by the studied LAB strain in WCJ were in the range of 1.05 to 1.67 , these values are higher than those reported in other studies for cabbage and other vegetable juice (Champagne et al., 2009; Savard et al., 2003). The higher growth of LAB observed in this study could be related to the nutritional content of Irish white cabbage. Among the LAB strains $L$. rhamnosus attained highest growth level $\left(\mathrm{OD}_{\max } 1.67\right)$ followed by L. plantarum $\left(\mathrm{OD}_{\max } 1.48\right)$ 
and L. brevis $\left(\mathrm{OD}_{\max }\right.$ 1.05). Though, $\mathrm{OD}_{\max }$ values were always lower in WCJ compared to MRS.

Generally, MRS medium has phosphate buffers, which are not available to WCJ, however, it is anticipated that higher $\mathrm{OD}_{\max }$ readings observed in MRS are due to a higher buffering capacity of MRS, rather than a nutrient deficiency of WCJ. Nonetheless, the results obtained in this study were encouraging as LAB grew satisfactory in the WCJ. Published literature shows that controlling the $\mathrm{pH}$ is critical in obtaining high biomass yields (Cerning et al., 1994). Therefore, in order to produce large volume (5 litre) of WCJ with high biomass, a further study was carried out in the bioreactor under controlled $\mathrm{pH}$ and dissolved oxygen.

\subsection{Fermentation under controlled conditions using 7 litre bioreactor}

\subsubsection{Growth of LAB during fermentation}

The time course for the growth of L. plantarum, L. rhamnosus and L. brevis is presented in Fig. 1. Total fermentation time ranged from $32-36 \mathrm{~h}$ and was dependent on the time of entering the decline phase for the LAB strains under study. However, all of the LAB strains showed different growth characteristics but reached the stationary phase within $24 \mathrm{~h}$ after which there was no significant increment observed in LAB growth. Initially, the concentration of probiotic bacteria was approximately $5 \log \mathrm{CFU} / \mathrm{ml}$; after $24 \mathrm{~h}$ of fermentation it increased to $9.19,9.47$ and 10.6 $\log \mathrm{CFU} / \mathrm{ml}$ for L. brevis, L. plantarum and L. rhamnosus, respectively. These results were in agreement with $\mathrm{OD}_{\max }$ data in the preliminary studies. It is anticipated that WCJ sustains the growth of probiotic bacteria due to protein content, total available sugars and micronutrients. In a previous report, it was found that cabbage is among the nutritionally well-balanced vegetables; it is rich in proteins, vitamins, carbohydrates, sugars (including fructose, glucose, sucrose and 
raffinose), essential n-3 fatty acid and linolenic acid (Batista et al., 2011). Thus, it is presumed that the bacteria utilized these nutrients as an energy source without additional nutrient supplementation. Growth of Lactobacillus in vegetable juices, including cabbage juice have been studied by various authors (Champagne et al., 2009; Gardner et al., 2001; Tolonen et al., 2004; Yoon et al., 2006) and maximum cell concentration has been reported to vary from $\log 7$ to 8 $\mathrm{CFU} / \mathrm{ml}$.

In a previous study, it was found that L. plantarum, L. casei and L. delbrueckii grew rapidly in sterilized cabbage juice without nutrient supplementation reaching nearly 8 log CFU/ml after 48 $\mathrm{h}$ of fermentation at $30^{\circ} \mathrm{C}$ (Yoon et al., 2006). Similarly, some other authors investigated the possibility of producing a functional carrot juice using L. rhamnosus and L. bulgaricus and a growth of $5 \times 10^{9} \mathrm{CFU} / \mathrm{ml}$ after $48 \mathrm{~h}$ was reported (Nazzaro et al., 2008). However, the results of the present study were encouraging as the time required to reach more than $9 \log \mathrm{CFU} / \mathrm{ml}$ viable cell count was shorter $(24 \mathrm{~h})$.

There are several models, which have been used to describe the sigmoid growth curve of a microorganism such as the Baranyi, Gompertz or Logistic models (Zwietering et al., 1990). Gompertz model is regarded as the most suitable to describe such microbial growth curves due to its simplicity and the low correlation, or interdependence, of the characteristic parameters. In this study, the lag phase duration as obtained from the Gompertz equation was minimal (less than 3 h) and was the magnitude of 1.27, 1.99 and 2.81 for L. plantarum L. brevis, and L. rhamnosus, respectively (Table 1). Among the probiotic cultures, L. rhamnosus and L. brevis grow at a significantly faster rate than $L$. plantarum (Table 1$)$. The specific growth rate $\left(\mu_{\max }\right)$ of $L$. rhamnosus and L. brevis was 1.5 times higher as compared to L. plantarum. The high growth 
rate resulted in a faster consumption of nutrients and thus the stationary phase for L. rhamnosus was attained in a total fermentation time of 16 h. L. brevis and L. rhamnosus showed 1.4 times higher specific growth rate $\left(\mu_{\max }\right)$ value as compared to L. plantarum.

Glucose was the main sugar present, accounting for $\approx 55 \%$ of the total sugar while fructose accounted for almost $45 \%$. This result is in line with previous findings, demonstrating that glucose and fructose are the main sugars present in white cabbage (Rosa et al., 2001). It is worth remarking that the sugar content reduced continuously as the bacterial population increased throughout the fermentation time. It was observed that fermentation led to a sharp depletion in the glucose and fructose levels in WCJ. Both the glucose and fructose were simultaneously consumed by the LAB strains during the fermentation process, but the rate of depletion was higher for glucose and more intense, which is usually because of the glucophilic nature of LAB. In the case of $L$. plantarum, after $24 \mathrm{~h}$ of fermentation only $33 \%$ residual glucose was left, while in the case of L. brevis it was $37 \%$ and for L. rhamnosus there was $10 \%$ remaining. A similar trend was also shown by fructose; however, the consumption was lower than glucose as after 24 h of fermentation only 76, 50 and $43 \%$ of fructose was consumed by L. plantarum L. brevis, and L. rhamnosus, respectively. As a result, the residual fructose concentrations were considerably higher than the residual glucose concentrations after fermentation.

\subsection{2 $\mathrm{pH}$ and organic acid production during fermentation}

Initially, the $\mathrm{pH}$ of the WCJ was 6.2 , which was adjusted to 7.0 before inoculation. As the fermentation was carried out under controlled $\mathrm{pH}$ conditions, further $\mathrm{pH}$ was adjusted by adding $1 \mathrm{M} \mathrm{HCL}$ or $4 \mathrm{M} \mathrm{KOH}$. The $\mathrm{pH}$ decreased slowly for the first 4 to $6 \mathrm{~h}$ of fermentation, and then dropped more quickly until LAB was in the decline phase. The drop in $\mathrm{pH}$ is due to the 
production of organic acids. Theoretically, the fermentation of 2 mol of glucose by LAB produces $3 \mathrm{~mol}$ of acetate and $2 \mathrm{~mol}$ of lactate via the fructose-6-phosphate shunt. The proportions of each metabolite depend on the carbon source; the strain used or the fermentation conditions. The presence of lactic and acetic acids in fermented foods is advantageous due to their antimicrobial property, thus preventing spoilage by other microorganisms.

Significant increment in acid concentration started after $8 \mathrm{~h}$ of fermentation and increased linearly until $24 \mathrm{~h}$, after which the production of acids was constant during the entire stationary phase. Lactic acid was the major end product of the WCJ fermentation followed by acetic acid, attaining the concentrations of $6.97,9.69$ and $12.2 \mathrm{~g} / \mathrm{l}$ lactic acid, while $0.45,0.59$ and $1.15 \mathrm{~g} / \mathrm{l}$ acetic acid were obtained for L. plantarum, L. rhamnosus and L. brevis, respectively. Absence of acetic acid results in a unilaterally sour taste with a flat and unusual aroma whereas its presence has a favourable effect on preservation. The presence of $0.51 \mathrm{~g} / \mathrm{l}$ citric acid was noted at the beginning of fermentation and its concentration reduced as the fermentation progressed. At the end of $24 \mathrm{~h}$ fermentation only $0.16,0.30$ and $0.45 \mathrm{~g} / \mathrm{l}$ citric acid was recorded for L. rhamnosus, L. plantarum and L. brevis, respectively.

The kinetic of lactic acid production was reasonably described by Mercier equation (Mercier et al., 1992) (Fig. 2) with a high coefficient of determination (Table 2). In the case of L. rhamnosus, the rate of production of lactic acid $\left(\mathrm{P}_{\mathrm{r}}\right)$ was $0.43 \mathrm{~h}^{-1}$, which was 1.72 and 2.15 times higher than that produced by L. brevis and L. rhamnosus, respectively. The numerical values of the parameter ' $\mathrm{P}_{0}$ ' were found to be close to zero, which is well in agreement with the obtained results. Similar results were also obtained for $L$. plantarum and L. brevis, but the rate of production of lactic acid was lower (41.7 and $53.5 \%$ for $L$. plantarum and $L$. brevis) than $L$. 
rhamnosus (Table 2). Some traces of citric and propionic acid was also observed although their concentration was not significant.

\subsubsection{The impact of fermentation on polyphenol content}

The major contribution to the AO capacity of plant foods is related to their content of polyphenols. Thus, it is important to consider the effect of fermentation on the TPC and TFC of the WCJ. Initially, the TPC of WCJ was in the range of 172.5 to $235.5 \mu \mathrm{g} \mathrm{GAE} / \mathrm{ml}$. Fermentation seemed to have a negative effect on the polyphenols content. In the case of $L$. plantarum and $L$. brevis, after $24 \mathrm{~h}$ of fermentation there was approx $15 \%$ reduction in TPC, while in the case of $L$. rhamnosus the loss was more prominent (24\%). The TFC of the fermented WCJ at the beginning of the fermentation existed in the range of 41.9-55.6 $\mu \mathrm{g} \mathrm{QE} / \mathrm{ml}$. A significant decrease in the TFC was seen as a result of LAB fermentation and approx 15.7, 21.7 and $23.9 \%$ losses in the TFC were recorded for L. brevis, L. plantarum and L. rhamnosus after $24 \mathrm{~h}$ of fermentation. These results were in agreement with the previously reported studies. Othman et al. (2009) reported a loss of 32-58\% in the polyphenols during spontaneous and controlled fermentations of "Chétoui" cultivar olives. Oseni and Akindahunsi (2011) also reported a 64\% reduction in the TPC during fermentation of Jatropha curcas and a similar trend was observed by Towo et al. (2006); these authors reported that LAB fermentation of cereal products leads to the reduction of phenolic compounds. LAB has a range of enzymes such as $\beta$-glucosidase, $\mathrm{p}$-coumaric acid decarboxylase, decarboxylase which may help in degrading certain phenolic compounds. However, results obtained in the present study were encouraging as LAB fermentation retained more than $75 \%$ of the initial polyphenols in the fermented WCJ. 
Degradation kinetics of the phytochemical content was modelled using zero-order (Eq. 4), firstorder (Eq. 5) and second-order kinetics model (Eq. 6). Coefficient of determination $\left(\mathrm{R}^{2}\right)$ was used as statistical measures for comparison of the experimental and model simulated values. First-order kinetics model fitted the experimental data with higher $\mathrm{R}^{2}$ value, ranging from 0.920.96 and 0.96-0.98 (Table 3) with a rate constant corresponding to 0.006-0.011 and 0.001-0.010 $\mathrm{h}^{-1}$ for TPC and TFC, respectively. The rate of TPC degradation was highest in L. rhamnosus fermented WCJ, which was 1.8 and 1.6 times higher than L. plantarum and L. brevis, respectively and a similar trend was observed for TFC where the rate of degradation was 1.7 and 2 times higher than L. plantarum and L. brevis, respectively.

\subsubsection{The impact of fermentation on antioxidant capacity}

The DPPH free radical scavenging capacity of the unfermented WCJ was in the range of 58.7$59.1 \mu \mathrm{g} \mathrm{AscE} / \mathrm{ml}$. In the case of WCJ fermented with L. plantarum, the loss of the DPPH radical scavenging was $\approx 13 \%$, while in the case of L. brevis and L. rhamnosus losses were 12 and 5\%, respectively, after $24 \mathrm{~h}$ of fermentation.

The ferric reducing AO potential of the fermented WCJ was evaluated for each strain as a function of incubation time. All the three LAB strain had similar trends during the fermentation period. The initial ferric reducing power WCJ expressed as an equivalent amount of trolox, decreased by the end of fermentation. After $24 \mathrm{~h}$ of fermentation, the reducing power decreased from 21.7 to $17.0 \mu \mathrm{g} \mathrm{TE} / \mathrm{ml}$ when WCJ was incubated with L. plantarum; from 15.8 to $13.9 \mu \mathrm{g}$ TE/ml with $L$. brevis, and from 15.0 to $13.7 \mu \mathrm{g} \mathrm{TE} / \mathrm{ml}$ when inoculated with $L$. rhamnosus. AO capacity depends on the structural conformation of phenolic compounds, and a similar trend was 
evident in this study. Thus, AO capacity is greatly influenced by the phenolic composition of the sample.

Degradation kinetics of the AO capacity was modelled by the application of zero-order (Eq. 4), first-order (Eq. 5) and second-order kinetics models (Eq. 6). The first-order kinetics model fitted the experimental data with a higher $\mathrm{R}^{2}$ value, ranging from $0.87-0.98$ and $0.96-0.98$ for $\mathrm{DPPH}$ radical scavenging capacity and FRAP, respectively (Table 4) with a rate constant corresponding to $0.002-0.005$ and $0.003-0.009 \mathrm{~h}^{-1}$ for DPPH radical scavenging capacity and FRAP, respectively. A different trend was observed in the case of AO capacity reduction, the maximum rate of reduction in DPPH scavenging capacity was evident in L. brevis and L. plantarum fermented WCJ which was 2.5 times higher than L. rhamnosus; however, no significant difference $(P>0.05)$ was observed between the rate of DPPH scavenging capacity reduction in L. brevis and L. plantarum fermented WCJ.

A similar pattern was observed for reduction in FRAP values, where L. brevis fermented WCJ showed 1.8 and 2.5 times higher rate of reduction for FRAP value. The differences in the trends between polyphenols and AO capacity could be understood by the fact that AO capacity of the food depends on the synergistic and redox interactions among the different compounds present in the vegetable. The reduction in one group of compounds may lead to the loss in functionality against certain type of free radicals without changing its functionality toward other radicals.

\subsubsection{Composition of probiotic cabbage juice}

The composition of food materials has an important role in maintaining prosperous health. Table 5 shows the nutritional composition of probiotic white cabbage juice. The fermented WCJ exhibited probiotic count in the range of 9.19 to $10.6 \log \mathrm{CFU} / \mathrm{ml}$. Protein concentration ranges 
from 15.8 to $17.4 \mathrm{mg} \mathrm{BSAE/} 1$ while carbohydrate content in terms of glucose is 2.16 to 3.33 $\mathrm{mg} / \mathrm{l}$ and fructose content ranges from 3.87 to 4.43 . Among the organic acids, LA content ranged from 6.97 to $12.2 \mathrm{~g} / \mathrm{l}$ while acetic acid content ranged from 0.45 to $1.15 \mathrm{~g} / \mathrm{l}$. Traces of citric acid was also noted; however, content was very low in the range of 0.16 to $0.45 \mathrm{~g} / \mathrm{l}$. Significant concentration of polyphenolic content was detected in the probiotic WCJ; which was in the range of 143.6 to $198.7 \mathrm{mg} \mathrm{GAE} / \mathrm{l}$.

\subsubsection{Shelf life analysis}

The probiotic WCJ was produced under the following conditions: an initial $\mathrm{pH}$ of 7.0, fermentation temperature at $37^{\circ} \mathrm{C}$, inoculated with $5 \%(\mathrm{v} / \mathrm{v})$ of inoculums size $(\approx 5 \log \mathrm{CFU} / \mathrm{ml})$ and fermented for a $24 \mathrm{~h}$ time period. There was a complete absence of any Enterobacteria or moulds in the fermented broth during the 30 days of storage at $4^{\circ} \mathrm{C}$. LAB counts in the probiotic WCJ were 9.19, 9.47 and $10.6 \log$ CFU/ml upon fermentation with L. brevis, L. plantarum and L. rhamnosus, respectively; similarly, $\approx 12.2,6.97$ and $9.69 \mathrm{~g} / \mathrm{l}$ LA content were produced upon fermentation with L. brevis, L. plantarum and L. rhamnosus, respectively. The stability of the probiotic bacteria during storage was monitored and a slight increase $(\approx 1 \log \mathrm{CFU} / \mathrm{ml})$ in the bacterial growth and lactic acid (1-2 g/l) was seen at the end of the 30 days of storage. The increase in LAB count could be due to the presence of residual glucose and fructose which was left after fermentation; thus, providing some essential growth nutrients. Pereira et al. (2011) observed in fermented cashew apple juice that up to 28 days of storage at $4^{\circ} \mathrm{C}$, there was slight increment in $L$. casei count and contentious decrease in $\mathrm{pH}$ which could be due to acid production. 
In order to exert the beneficial effects of probiotic foods, a minimum probiotic therapeutic daily dose of 8 to $9 \log$ CFU has been proposed, which corresponds to a daily intake of $100 \mathrm{~g}$ of a food product containing $6 \log$ up to $7 \log$ CFU/g (Lee and Salminen, 1995), which this product meets. Guo et al. (2009) reported that the $\mathrm{pH}$ of $L$. casei fermented milk was initially 5.59 and reduced to 4.60 during storage. In addition, $L$. case $i$ was capable in producing acid even at refrigerated temperatures which in line with that observed in this study. However, no significant changes in the TPC, TFC value and AO capacity were observed during storage.

\section{Conclusion}

WCJ was used as a sole source of nutrition for probiotic fermentation and was found to support prolific growth of L. brevis, L. plantarum and L. rhamnosus. The model equations used herein allowed accurate description of the microbial kinetics and characterization of the main products. Furthermore, the change in the response of a variable with respect to time was described. The parameters thus obtained were very useful for understanding the culture dynamics such as the rate by which a particular variable was increased/reduced or produced. All of the LAB cultures survived throughout the 30 days of refrigerated storage without substantial viability losses, phytochemical content or AO capacity loss. From the results of this study, it is concluded that probiotic WCJ could be served as a good healthy substitute of functional foods containing probiotics for vegetarians and consumers who have allergies to dairy products. Furthermore, vegetables based probiotic products would offer bioactive characteristics which are lacking in typical dairy based probiotic products. Complementary studies on the impact of the fermentation process on sensory acceptance, aroma and on the nutritional value such as vitamins and other functional properties are considered as a relevant avenue for further studies. 


\section{Acknowledgment}

The authors would like to gratefully acknowledge Dr. Shilpi Gupta for technical assistance and funding from the Irish Government under the Technological Sector Research Scheme (Strand III) of the National Development Plan.

\section{References}

Agerholm-Larsen, L., Raben, A., Haulrik, N., Hansen, A., Manders, M., Astrup, A., 2000. Effect of 8 week intake of probiotic milk products on risk factors for cardiovascular diseases. Eur. J. Clin. Nutr. 54, 288.

Batista, C., Barros, L., Carvalho, A.M., Ferreira, I.C.F.R., 2011. Nutritional and nutraceutical potential of rape (Brassica napus var napus) and" tronchuda" cabbage (Brassica oleraceae var costata) inflorescences. Food Chem. Toxicol. 49, 1208-1214

Beganović, J., Pavunc, A.L., Gjuračić, K., Špoljarec, M., Šušković, J., Kos, B., 2011. Improved sauerkraut production with probiotic strain Lactobacillus plantarum L4 and Leuconostoc mesenteroides LMG 7954. J. Food Sci. 76, M124-M129.

Bradford, M.M., 1976. A rapid and sensitive method for the quantitation of microgram quantities of protein utilizing the principle of protein-dye binding. Anal. Biochem. 72, 248-254.

Biazar, J., Tango, M., Babolian, E., Islam, R., 2003. Solution of the kinetic modeling of lactic acid fermentation using Adomian decomposition method. Appl. Math. Comput. 144, 433439.

Cerning, J., Renard, C., Thibault, J., Bouillanne, C., Landon, M., Desmazeaud, M., Topisirovic, L., 1994. Carbon source requirements for exopolysaccharide production by Lactobacillus casei CG11 and partial structure analysis of the polymer. Appl. Environ. Microbiol. 60, 39143919. 
Champagne, C.P., Savard, T., Barrette, J., 2009. Production of lactic acid bacteria on spent cabbage juice. Int. J. Food Agric. Environ. 7, 82-87.

de Vrese, M., Stegelmann, A., Richter, B., Fenselau, S., Laue, C., Schrezenmeir, J., 2001. Probiotics-compensation for lactase insufficiency. Am. J. Clin. Nutr. 73, 421S-429S.

Gardner, N.J., Savard, T., Obermeier, P., Caldwell, G., Champagne, C.P., 2001. Selection and characterization of mixed starter cultures for lactic acid fermentation of carrot, cabbage, beet and onion vegetable mixtures. Int. J. Food Microbiol. 64, 261-275.

Gotcheva, V., Hristozova, E., Hristozova, T., Guo, M., Roshkova, Z., Angelov, A., 2002. Assessment of potential probiotic properties of lactic acid bacteria and yeast strains. Food Biotechnol. 16, 211-225.

Guo, Z., Wang, J., Yan, L., Chen, W., Liu, X., Zhang, H., 2009. In vitro comparison of probiotic properties of Lactobacillus casei Zhang, a potential new probiotic, with selected probiotic strains. LWT-Food Sci. Technol. 42, 1640-1646.

Jaiswal, A.K., Gupta, S., Abu-Ghannam, N., 2012a. Optimisation of lactic acid fermentation of York cabbage for the development of potential probiotic products. Int. J. Food Sci. Technol. $47,1605-1612$.

Jaiswal, A.K., Rajauria, G., Abu-Ghannam, N., Gupta, S., 2012b. Effect of different solvents on polyphenolic content, antioxidant capacity and antibacterial activity of Irish York cabbage. J. Food Biochem. 36, 344-358.

Khan, R.S., Grigor, J., Winger, R., Win, A., 2013. Functional food product development Opportunities and challenges for food manufacturers. Trends Food Sci. Technol. 30, 27-37.

Kusznierewicz, B., Smiechowska, A., Bartoszek, A., Namiesnik, J., 2008. The effect of heating and fermenting on antioxidant properties of white cabbage. Food Chem. 108, 853-861. 
Lee, Y.K., Salminen, S., 1995. The coming of age of probiotics. Trends Food Sci. Technol. 6, $241-245$.

Mercier, P., Yerushalmi, L., Rouleau, D., Dochain, D., 1992. Kinetics of lactic acid fermentation on glucose and corn by Lactobacillus amylophilus. J. Chem. Technol. Biot. 55, 111-121.

Nazzaro, F., Fratianni, F., Sada, A., Orlando, P., 2008. Synbiotic potential of carrot juice supplemented with Lactobacillus spp. and inulin or fructooligosaccharides. J. Sci. Food Agr. $88,2271-2276$.

Nomoto, K., 2005. Prevention of infections by probiotics. J. Biosci. Bioeng. 100, 583-592.

Oseni, O., Akindahunsi, A., 2011. Some phytochemical properties and effect of fermentation on the seed of Jatropha curcas L. Am. J. Food Technol. 6, 158-165.

Othman, N.B., Roblain, D., Chammen, N., Thonart, P., Hamdi, M., 2009. Antioxidant phenolic compounds loss during the fermentation of Chétoui olives. Food Chem. 116, 662-669.

Pereira, A.L.F., Maciel, T.C., Rodrigues, S., 2011. Probiotic beverage from cashew apple juice fermented with Lactobacillus casei. Food Res. Int. 44, 1276-1283.

Rajauria, G., Jaiswal, A.K., Abu-Gannam, N., Gupta, S., 2012. Antimicrobial, antioxidant and free radical-scavenging capacity of brown seaweed Himanthalia elongata from western coast of Ireland. J. Food Biochem. 37, 322-335.

Rosa, E., David, M., Gomes, M.H., 2001. Glucose, fructose and sucrose content in broccoli, white cabbage and Portuguese cabbage grown in early and late seasons. J. Sci. Food Agric. $81,1145-1149$.

Savard, T., Gardner, N., Champagne, C., 2003. Growth of Lactobacillus and Bifidobacterium cultures in a vegetable juice medium, and their stability during storage in a fermented vegetable juice. Sci. Des. Alim. 23, 273-283. 
Sindhu, S.C., Khetarpaul, N., 2003. Effect of feeding probiotic fermented indigenous food mixture on serum cholesterol levels in mice. Nutr. Res. 23, 1071-1080.

Tolonen, M., Rajaniemi, S., Pihlava, J.M., Johansson, T., Saris, P., Ryhänen, E.L., 2004. Formation of nisin, plant-derived biomolecules and antimicrobial activity in starter culture fermentations of sauerkraut. Food Microbiol. 21, 167-179.

Towo, E., Matuschek, E., Svanberg, U., 2006. Fermentation and enzyme treatment of tannin sorghum gruels: effects on phenolic compounds, phytate and in vitro accessible iron. Food Chem. 94, 369-376.

Yoon, K.Y., Woodams, E.E., Hang, Y.D., 2006. Production of probiotic cabbage juice by lactic acid bacteria. Bioresource Technol. 97, 1427-1430.

Zwietering, M., Jongenburger, I., Rombouts, F., Van't Riet, K., 1990. Modeling of the bacterial growth curve. Appl. Environ. Microbiol. 56, 1875-1881. 


\section{Figure Captions}

Fig. 1. Time course for the growth of L. plantarum, L. rhamnosus and L. brevis in WCJ

Fig. 2. Time course for the production of lactic acid by L. plantarum, L. rhamnosus and L. brevis in WCJ 
Table 1. Value of parameters obtained by non-linear regression of Gompertz equation for the growth of probiotic cultures under study

\begin{tabular}{lllll}
\hline & \multicolumn{4}{c}{$\mathrm{CFU} / \mathrm{ml}$} \\
\cline { 2 - 5 } & $\mathrm{A}$ & $\mu_{\max }\left(\mathrm{h}^{-1}\right)$ & $\lambda(\mathrm{h})$ & $\mathrm{R}^{2}$ \\
\hline L. plantarum & 4.72 & 0.24 & 1.27 & 0.9633 \\
& $(4.13-5.32)$ & $(0.15-0.32)$ & $(-2.39-4.93)$ & \\
\hline L. rhamnosus & 5.88 & 0.36 & 2.81 & 0.9730 \\
& $(3.84-7.91)$ & $(0.15-0.58)$ & $(-1.47-7.08)$ & \\
\hline L. brevis & 4.31 & 0.33 & 1.99 & 0.9669 \\
& $(3.62-5.00)$ & $(0.14-0.51)$ & $(-1.75-5.74)$ & \\
\hline
\end{tabular}

Values in brackets are the confidence intervals

A: log increase in population; $\mu_{\max }$ maximum specific growth rate of cells; $\lambda$ : lag phase 
Table 2. Value of parameters obtained by non-linear regression of Mercier equation for lactic acid production by the probiotic cultures under study

\begin{tabular}{lllll}
\hline & $\mathrm{P}_{0}(\mathrm{~g} / \mathrm{l})$ & $\mathrm{P}_{\mathrm{m}}(\mathrm{g} / \mathrm{l})$ & $\mathrm{P}_{\mathrm{r}}\left(\mathrm{h}^{-1}\right)$ & $\mathrm{R}^{2}$ \\
\hline L. plantarum & 0.08 & 8.90 & 0.25 & 0.998 \\
& $(0.04-0.12)$ & $(8.51-9.29)$ & $(0.22-0.28)$ & \\
\hline L. rhamnosus & 0.01 & 12.57 & 0.43 & 0.998 \\
& $(0.01-0.03)$ & $(11.95-13.18)$ & $(0.35-0.51)$ & \\
\hline L. brevis & 0.00 & 9.81 & 0.20 & 0.914 \\
& $(0.00-0.04)$ & $(7.67-12.0)$ & $(0.40-0.10)$ & \\
& & & &
\end{tabular}

Values in brackets are the confidence intervals

$P_{o}$ : initial lactic acid concentration; $P_{m}$ : maximum concentration of lactic acid;

$P_{r}$ : ratio between the initial volumetric rate of product formation $\left(r_{p}\right)$ and the

initial product concentration $\left(P_{o}\right)$ 
Table 3. Kinetics parameters for phytochemical content of WCJ during fermentation

\begin{tabular}{ccccccccccc}
\hline & \multicolumn{3}{c}{ Zero-order kinetic } & \multicolumn{3}{c}{ First-order kinetic } & \multicolumn{3}{c}{ Second-order kinetic } \\
\cline { 2 - 10 } & $\mathrm{C}_{0}$ & $k\left(\mathrm{~h}^{-1}\right)$ & $\mathrm{R}^{2}$ & $\mathrm{C}_{0}$ & $k\left(\mathrm{~h}^{-1}\right)$ & $\mathrm{R}^{2}$ & $\mathrm{C}_{0}$ & $k\left(\mathrm{~h}^{-1}\right)$ & $\mathrm{R}^{2}$ \\
\hline L. plantarum & & & & & & & & & \\
TPC & 170.7 & 0.910 & 0.92 & 171.6 & 0.006 & 0.93 & 172.5 & 0.0001 & 0.91 \\
TFC & 42.6 & 0.352 & 0.97 & 42.9 & 0.001 & 0.98 & 42.7 & 0.0003 & 0.97 \\
L. rhamnosus & & & & & & & & & \\
TPC & 188.5 & 1.744 & 0.92 & 190.9 & 0.011 & 0.94 & 190.2 & 0.0001 & 0.94 \\
TFC & 40.7 & 0.336 & 0.95 & 41.1 & 0.010 & 0.97 & 41.0 & 0.0003 & 0.96 \\
L. brevis & & & & & & & & & \\
TPC & 236.8 & 1.429 & 0.96 & 238.2 & 0.007 & 0.97 & 238.8 & 0.0001 & 0.94 \\
TFC & 56.1 & 0.400 & 0.96 & 56.2 & 0.005 & 0.97 & 56.8 & 0.0002 & 0.94 \\
\hline
\end{tabular}


Table 4. Kinetics parameters for antioxidant capacity of WCJ during fermentation

\begin{tabular}{ccccccccccc}
\hline & \multicolumn{3}{c}{ Zero-order kinetic } & \multicolumn{2}{c}{ First-order kinetic } & \multicolumn{3}{c}{ Second-order kinetic } \\
\cline { 2 - 10 } & $\mathrm{C}_{0}$ & $k\left(\mathrm{~h}^{-1}\right)$ & $\mathrm{R}^{2}$ & $\mathrm{C}_{0}$ & $k\left(\mathrm{~h}^{-1}\right)$ & $\mathrm{R}^{2}$ & $\mathrm{C}_{0}$ & $k\left(\mathrm{~h}^{-1}\right)$ & $\mathrm{R}^{2}$ \\
\hline L. plantarum & & & & & & & & & \\
DPPH & 57.7 & 0.288 & 97.5 & 57.9 & 0.005 & 97.6 & 58.1 & 0.0001 & 97.2 \\
FRAP & 15.7 & 0.069 & 96.6 & 15.7 & 0.005 & 96.9 & 15.8 & 0.003 & 95.3 \\
L. rhamnosus & & & & & & & & & \\
DPPH & 59.2 & 0.110 & 84.4 & 59.1 & 0.002 & 87.4 & 59.3 & 0.00003 & 84.1 \\
FRAP & 15.0 & 0.041 & 97.6 & 15.0 & 0.003 & 97.6 & 15.0 & 0.0002 & 95.5 \\
L. brevis & & & & & & & & & \\
DPPH & 57.6 & 0.251 & 86.3 & 57.7 & 0.005 & 87.1 & 56.8 & 0.0001 & 82.2 \\
FRAP & 21.0 & 0.166 & 95.1 & 21.2 & 0.009 & 96.2 & 21.2 & 0.0005 & 96.4 \\
\hline
\end{tabular}

$C_{0}$ Model estimated value, $k$ reaction rate constant 
Table 5. Nutritional composition of probiotic cabbage juice

\begin{tabular}{cccc}
\hline & \multicolumn{2}{c}{ LAB strain used for juice preparation } \\
\cline { 2 - 4 } & L. brevis & L. plantarum & L. rhamnosus \\
\hline Probiotic count (log CFU/ml) & $9.19 \pm 0.24$ & $9.47 \pm 0.15$ & $10.6 \pm 0.23$ \\
Protein content (mg BSAE/l) & $16.4 \pm 0.73$ & $15.8 \pm 1.46$ & $17.4 \pm 0.30$ \\
Carbohydrate content & & & \\
Glucose (mg/l) & $3.33 \pm 0.29$ & $2.31 \pm 0.17$ & $2.16 \pm 0.22$ \\
Fructose (mg/l) & $3.87 \pm 0.26$ & $4.41 \pm 0.15$ & $4.43 \pm 0.13$ \\
Organic acid content & & & \\
Lactic acid (g/l) & $12.2 \pm 0.18$ & $6.97 \pm 0.29$ & $9.69 \pm 0.01$ \\
Acetic acid (g/l) & $1.15 \pm 0.11$ & $0.45 \pm 0.07$ & $0.59 \pm 0.12$ \\
Citric acid (g/l) & $0.16 \pm 0.01$ & $0.30 \pm 0.05$ & $0.45 \pm 0.09$ \\
Polyphenolic content & & & \\
TPC (mg GAE/l) & $198.7 \pm 2.89$ & $148.6 \pm 1.32$ & $143.6 \pm 1.75$ \\
TFC (mg QE/l) & $46.9 \pm 1.24$ & $35.3 \pm 1.54$ & $31.9 \pm 1.02$ \\
Antioxidant capacity & & & \\
FRAP value (mg TE/l) & $13.9 \pm 0.02$ & $17.0 \pm 0.12$ & $13.7 \pm 0.17$ \\
DPPH RSA (mg AAE/l) & $51.9 \pm 0.45$ & $51.1 \pm 0.79$ & $56.1 \pm 0.32$ \\
\hline
\end{tabular}

LAB, Lactic acid bacteria; BSA, Bovine serum albumin; TPC, Total phenolic content; GAE, Gallic acid equivalent; TFC, Total flavonoid content; QE, Quercetin equivalent; TE, Trolox equivalent; AAE, Ascorbic acid equivalent; FRAP, Ferric reducing antioxidant potential; RSA, Radical scavenging capacity 


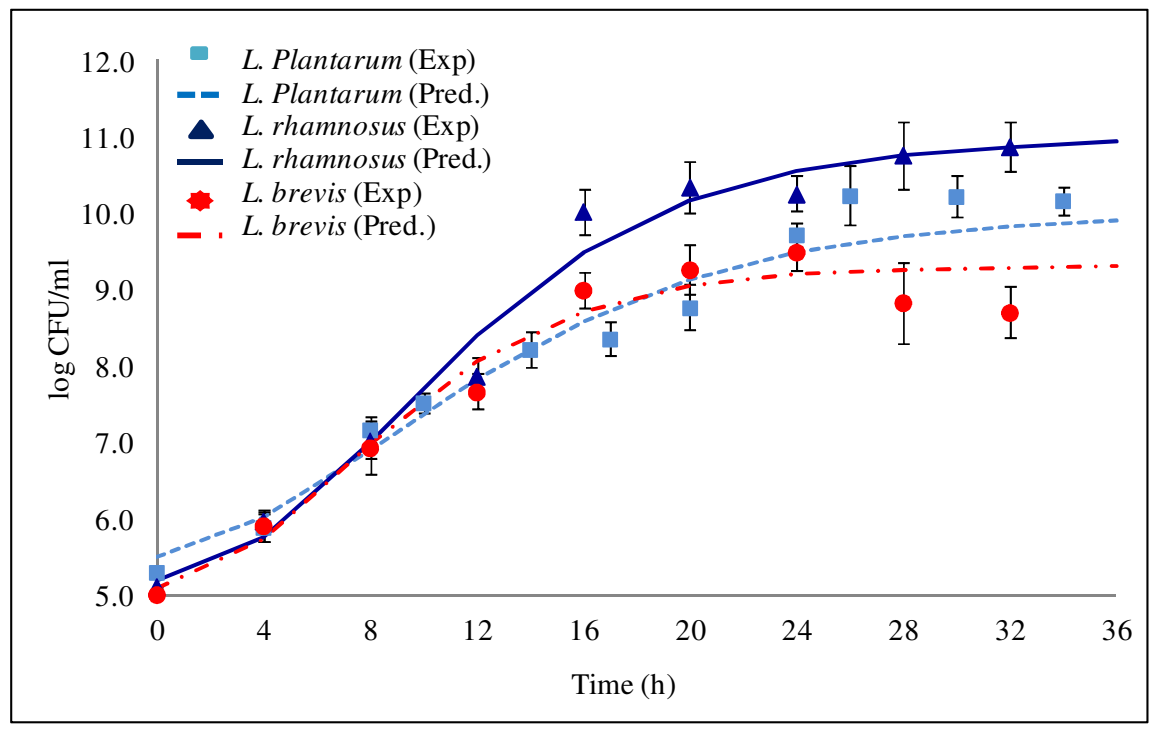

Fig. 1. Time course for the growth of L. plantarum, L. rhamnosus and L. brevis in WCJ 


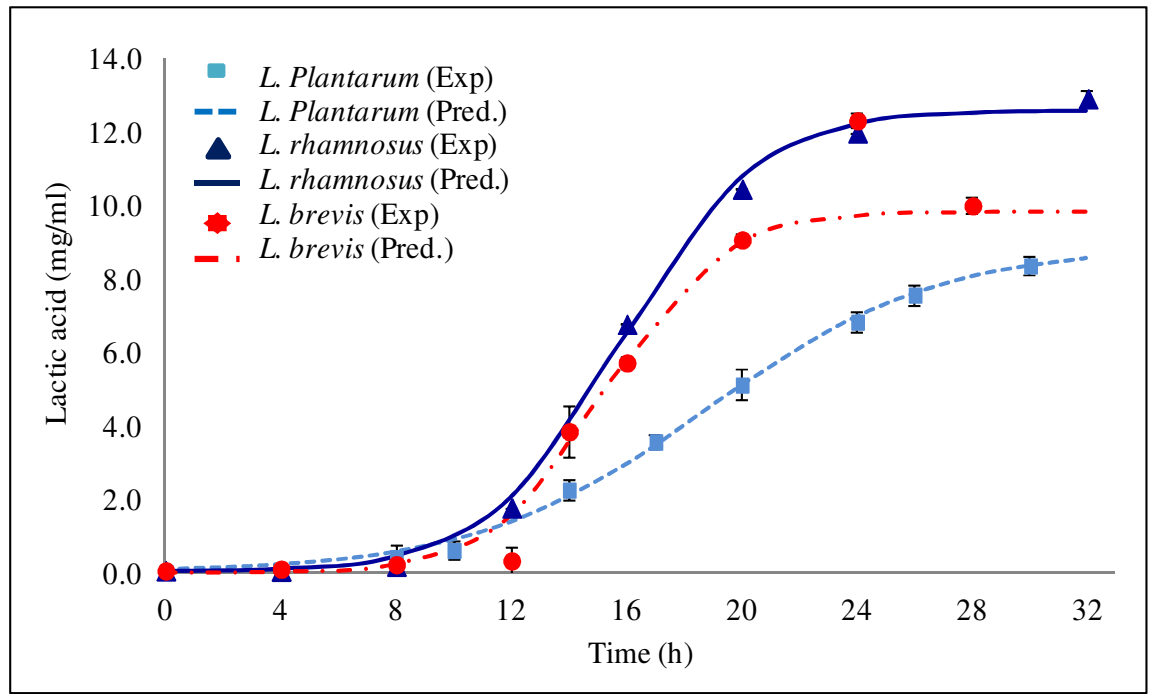

Fig. 2. Time course for the production of lactic acid by L. plantarum, L. rhamnosus and L. brevis in WCJ 